\title{
Modeling of Instantaneous Passive Pitch of Flexible Flapping Wings
}

\author{
Chang-kwon Kang ${ }^{1 *}$ and Wei Shyy ${ }^{2 \dagger}$ \\ * Department of Aerospace Engineering, University of Michigan, Ann Arbor, MI, 48109 \\ ${ }^{\dagger}$ Department of Mechanical Engineering, Hong Kong University of Science and Technology, Clear Water Bay, \\ Hong Kong
}

While the biological flyers often showcase high deformed wing structures, the effects of flexibility on the flapping wing aerodynamics remain inadequately understood. A major challenge in the study of flexible flapping wings is that the resulting wing motion is an outcome of a dynamic balance between the structural dynamics of the wing and the unsteady aerodynamics. Since the effective angle of attack is affected by structural deformation, the wing resulting kinematics is a priori unknown. In this study, we solve for the temporal evolution of the wing deformation by modeling the wing as an elastic beam under an imposed nominal kinematics. We use the Morison equation, which consists of the added mass and aerodynamic damping forces, for the fluid dynamic force term. The resulting wing deformation is correlated with a numerical solution of fully coupled aeroelastic framework. While the end-of-the-stroke passive pitch angle agrees well with the numerical solution, the midstroke pitch angle needs to be empirically corrected. Based on these two angles, we construct a first-order harmonic passive pitch motion. The derived model can satisfactorily predict the phase lag and reasonably the angular amplitude. The current results can be a stepping stone toward formulation of a quick predictive model for instantaneous aerodynamic forces on a flexible flapping wing, for bio-flight and human engineered MicroAir Vehicles.

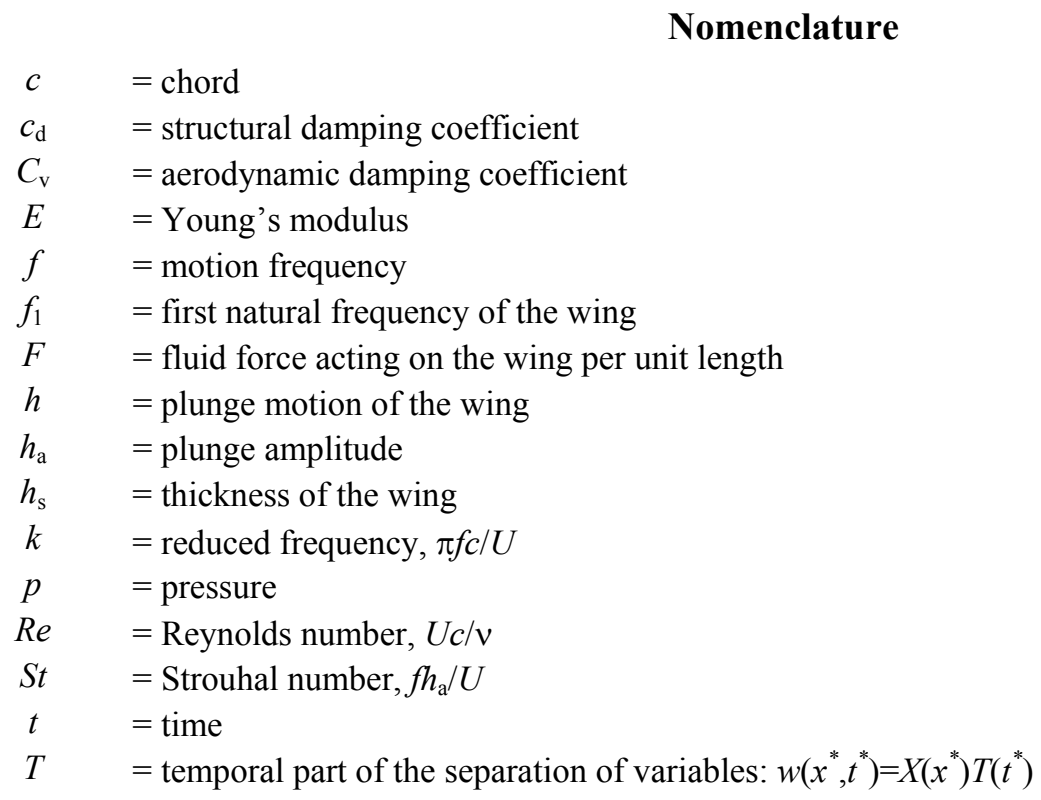

${ }^{1}$ Postdoctoral Research Fellow, Department of Aerospace Engineering, University of Michigan, AIAA Member.

${ }^{2}$ Provost \& Chair Professor, Department of Mechanical Engineering, Hong Kong University of Science and Technology, AIAA Fellow. 
$U \quad=$ reference velocity: $2 \pi f h_{\mathrm{a}}$ for hover $\quad[\mathrm{m} / \mathrm{s}]$

$u \quad=$ velocity $\quad[\mathrm{m} / \mathrm{s}]$

$v \quad=$ wing displacement: $v=w+h \quad$ [m]

$X \quad=$ spatial part of the separation of variables: $w\left(x^{*}, t^{*}\right)=X\left(x^{*}\right) T\left(t^{*}\right)$

$w \quad=$ wing deformation relative to the imposed motion $h \quad$ [m]

$\alpha \quad=$ passive pitch angle $\quad\left[{ }^{\circ}\right]$

$\alpha_{\mathrm{a}} \quad=$ angular amplitude $\quad\left[{ }^{\circ}\right]$

$\alpha_{\mathrm{m}} \quad=$ midstroke passive pitch angle $\quad\left[{ }^{\circ}\right]$

$\alpha_{e} \quad=$ end-of-the-stroke passive pitch angle $\quad\left[{ }^{\circ}\right]$

$\beta \quad=$ coefficient for the aerodynamic damping term

$\gamma \quad=$ non-dimensional tip deformation parameter: $2\left(1+\pi / 4 \rho^{*} h_{\mathrm{s}}{ }^{*}\right) /\left\{k\left(f_{1}^{2} / f^{2}-1\right)\right\}$

$\delta \quad=$ coefficient for the acceleration related term

$\zeta=$ coefficient for the structural damping term

$\varphi \quad=$ phase lag between rotational and translational motion $\quad\left[{ }^{\circ}\right]$

$v \quad=$ kinematic viscosity of fluid $\quad\left[\mathrm{m}^{2} / \mathrm{s}\right]$

$\Pi_{0} \quad=$ effective inertia: $\rho^{*} h_{\mathrm{s}}{ }^{*}(k / \pi)^{2}$

$\Pi_{1} \quad=$ effective stiffness: $E h_{\mathrm{s}}{ }^{* 3} /\left(12 \rho_{\mathrm{f}} U^{2}\right)$

$\rho_{\mathrm{f}} \quad=$ density of fluid $\quad\left[\mathrm{kg} / \mathrm{m}^{3}\right]$

$\rho_{\mathrm{s}} \quad=$ density of structure $\quad\left[\mathrm{kg} / \mathrm{m}^{3}\right]$

$\omega_{\mathrm{f}} \quad=$ non-dimensional natural frequency of the wing: $2 \pi f_{1} / f$

$(\cdot)^{*} \quad=$ variables normalized either by $c$ (length), $1 / f($ time $)$, or $\rho_{\mathrm{f}}$ (density) [1]

\section{Introduction}

Scaling laws clearly indicate that Micro-Air Vehicles (MAVs) cannot utilize the same lift generation mechanisms $S_{\text {as larger aircrafts }}{ }^{1}$. Instead, moving wings are needed to conduct flight mission ${ }^{1}$. These flyers can benefit from active $^{2}$ and passive ${ }^{3}$ control of wing kinematics for performance enhancement. However, since MAVs are light and operate at low speeds, they are sensitive to wind gust. Moreover, the wings are often flexible and may substantially deform during flight. As a result, the aerodynamics, structural dynamics, and flight dynamics are closely linked to each other. Wing motions affect the fluid force on the wing, which in turn leads to changes in the wing shapes and motions. These highly coupled nonlinearities make the successful design of MAVs challenging.

On the other hand, biological flyers showcase the desirable flight characteristics and performance objectives that can be applied to the design of MAVs ${ }^{1}$. Using flapping wings, these flyers exhibit rapid accelerations and decelerations in small confined spaces. Furthermore, they are quickly able to respond to various environmental changes including wind gusts and threat avoidance ${ }^{1}$.

Birds, bats, and insects possess flexible wings that experience large deformations during flight ${ }^{4-6}$. Compliance structures are not uncommon in nature and can be beneficial for their survival ${ }^{7,8}$. A locust ${ }^{4}$ or a hawkmoth ${ }^{9}$ also use their flexible wings to improve the aerodynamic performance. However, our understanding of the closely coupled fluid-structure flapping wing systems has been incomplete to explain all the salient features of the biological flight and to be applied to the development of MAVs.

One of the main challenges of the analysis of flexible flapping wing aerodynamics is that the wing kinematics is not known a priori. The angle of attack is affected by the structural deformation as a result of dynamic balance between the wing inertial force, elastic restoring force, and fluid dynamic forces. A popular approach to overcome this challenge is to measure the wing kinematics of biological flyers using a high speed camera and explore the aerodynamics based on these observations ${ }^{4,10,11}$. This way one can circumvent the modeling of the wing structure, which can be intriguingly complex. Alternatively, one can use an abstracted model by simplifying the wing structure, geometry, and kinematics to illuminate the specific phenomena in detail. A merit of this approach is that the interplay between the wing deformation and the aerodynamics can be highlighted. For example, scaling relationships could be derived for between aerodynamic performance and a dimensionless parameter that depends on a priori known parameters ${ }^{12}$. 
Such scaling relationships are useful for the preliminary design of MAVs. Specifically, these relationships can be used to estimate the orders of magnitudes of the expected propulsive force or efficiency as a function of the structural parameters and motion kinematics. However, they are measured in time-averaged quantities ${ }^{12}$. To model the flight dynamics for the analysis of stability and control of a flight vehicle, aerodynamic forces need to be formulated as a function of time, which requires a model for the instantaneous wing movement.

The objective of this study is to establish a framework to analyze and to estimate the instantaneous wing deformations. We consider a passive pitching, active plunge motion of a two-dimensional wing in hover at $R e=U c / v=100$, based on the midstroke velocity $U$, wing chord length $c$, and kinematic viscosity of the fluid $v$. We model the wing as a linear elastic flat plate and the fluid forces using the Morison equation ${ }^{13}$, modeling for the added mass and aerodynamic damping forces at low Reynolds numbers. We correlate the obtained formulation to the numerical data, which are computed using a fully-coupled Navier-Stokes aeroelastic solver ${ }^{14}$. Finally, we construct the instantaneous passive pitch using the derived formulation and compare to the time history of the numerical computed passive pitch.

\section{Methodology}

\section{A. Governing Equations for the Fluid-Structure Interaction System}

We consider a non-dimensional flow field with unit density initiated by a hovering two-dimensional flat plate with unit chord and flat edges ${ }^{14}$. A sinusoidal plunge motion $h$ with amplitude $h_{\mathrm{a}}$ and frequency $f$ is imposed on the leading-edge (LE) of the wing as

$$
h(t)=h_{\mathrm{a}} \cos (2 \pi f t)
$$

as a function of time $t$, see also Fig. 1. In the absence of freestream for hovering wings, we set the maximum translational velocity $U$ of the flat plate at the $\mathrm{LE}$ as the reference velocity, such that $U=2 \pi f h_{\mathrm{a}}{ }^{14-16}$. Note that reduced frequency in hover becomes a geometric quantity: $k=\pi f_{c} / U=c /\left(2 h_{\mathrm{a}}\right)$. The Strouhal number, another important parameter in flapping wing aerodynamics, becomes a constant for hovering motions: $S t=f h_{\mathrm{a}} / U=1 /(2 \pi)$.

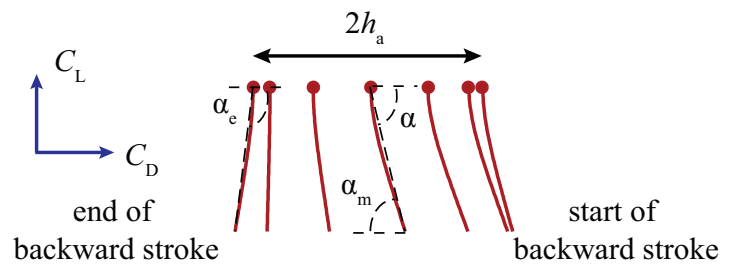

Figure 1. Schematic of the wing motion with amplitude $h_{\mathrm{a}}$. Wing deformations lead to a passive pitch angle $\alpha$. The angles at the mid and end-of-the-strokes are $\alpha_{\mathrm{m}}$ and $\alpha_{\mathrm{e}}$, respectively. The directions of the lift and drag are normal and parallel to the imposed plunge motion, respectively. Leading-edge of the wing is represented with a red dot.

The flow field is governed by the unsteady Navier-Stokes equations with constant density $\rho_{\mathrm{f}}$ and viscosity

$$
\begin{gathered}
\nabla^{*} \cdot \boldsymbol{u}^{*}=0 \\
\frac{\partial \boldsymbol{u}^{*}}{\partial t^{*}}+\boldsymbol{u}^{*} \cdot \nabla^{*} \boldsymbol{u}^{*}=-\nabla^{*} p^{*}+\frac{1}{R e} \Delta^{*} \boldsymbol{u}^{*}
\end{gathered}
$$

for the velocity $\boldsymbol{u}$, pressure $p$, and time $t$. The superscript $\left(^{*}\right)$ indicates non-dimensional variables. The dimensional variables are non-dimensionalized with a reference velocity $U$, inverse of the motion frequency $1 / f$, and chord $c$, respectively. 
The wing is an elastic flat plate of uniform thickness $h_{\mathrm{s}}$, density $\rho_{\mathrm{s}}$, and Young's modulus $E$. As the flat plate follows the imposed horizontal motion, Eq. (1), at the LE, the resulting fluid dynamic force dynamically balances with the wing inertia and the elastic bending forces, modeled locally as a linear Euler-Bernoulli beam as

$$
\Pi_{0} \frac{\partial^{2} v^{*}}{\partial t^{* 2}}+c_{\mathrm{d}} \frac{\partial v^{*}}{\partial t^{*}}+\Pi_{1} \Delta^{* 2} v^{*}=F^{*}
$$

where $v$ is the displacement due to bending motion, $\Pi_{0}=\rho^{*} h_{\mathrm{s}}{ }^{*}(k / \pi)^{2}$ is the effective inertia, the inertia of the wing normalized by the fluid dynamic variables ${ }^{12}, \rho^{*}=\rho_{\mathrm{s}} / \rho_{\mathrm{f}}$ is the density ratio between the wing density and the fluid density, $c_{\mathrm{d}}$ is the non-dimensional structural damping coefficient, $\Pi_{1}=E h_{\mathrm{s}}{ }^{* 3} /\left(12 \rho_{\mathrm{f}} U^{2}\right)$ is the effective stiffness, the wing stiffness normalized by the fluid dynamic variables ${ }^{12}$, and $F$ is the distributed transverse fluid force per unit length on the wing, such that $F^{*}=F /\left(\rho_{\mathrm{f}} U^{2}\right)$. The resulting wing camber deformations $w=v-h$ can also be regarded as a pitch rotation $\alpha\left(t^{*}\right)$, the angle between the trailing-edge (TE) and LE.

\section{B. Case Setup}

We compare the current analysis with the results from Navier-Stokes equation computations fully coupled to a structural dynamics solver ${ }^{14}$. For the numerical computations, the Reynolds number is $R e=100$, relevant to a fruit fly. The density ratio is $\rho^{*}=7.8$ and the thickness ratio is $h_{\mathrm{s}}{ }^{*}=0.02$. The reduced frequency $0.25<k<3.75$ is motivated from the selection of $h_{\mathrm{a}}$ of biological relevance. The frequency ratio is in the range of $0.06<f l f_{1}<0.82$, motivated from the previous findings that the natural flyers operate at a frequency ratio less than $0.8^{17}$. The natural frequency $f_{1}$ is measured in the chordwise direction between the LE and TE. More details can be found in Kang and Shyy ${ }^{14}$.

\section{Results and Discussion}

\section{A. Derivation of the Response of the Wing Trailing-edge}

The fluid flow and the wing displacement simultaneously satisfy Eqs. (2) and (3), the Navier-Stokes equation and the beam equation, respectively. To model the dynamics of the structural response, we analyze the physics based Eq. (3) with simplifying approximations for the fluid dynamic force $F^{*}$. The Navier-Stokes equation is nonlinear in the convection term and the full explicit expression for $F^{*}$ is not available.

The total fluid dynamic force consists of two terms, i.e. the added mass force and the force induced by the vorticity in the flow field ${ }^{12,18}$. The added mass force is caused by the acceleration of the wing and is linearly proportional to the wing acceleration in the linearized aerodynamics theories. With increasing $k$, the added mass force gains its relative contribution to the total fluid dynamic force ${ }^{14}$.

Based on scaling arguments, we found that, for the deformation of flexible flapping wings, the fluid dynamics force term could be well approximated by the added mass force for various cases at $k>O(1)$ and $R e>O\left(10^{2}\right)^{12}$. Moreover, based on Eq. (3) we derived scaling relationships between the aerodynamic performance, such as the time-averaged propulsive force and the propulsive efficiency, and the relative shape deformation parameter $\gamma$ defined as

$$
\gamma=\frac{S t k\left(1+\frac{4}{\pi} \rho^{*} h_{s}^{*}\right)}{\Pi_{0}\left(f_{1}^{2} / f^{2}-1\right)} .
$$

For the current parameter selection, the resulting $\gamma$, given by Eq. (4), would be equivalent to that of a fruit fly ${ }^{14}$.

In order to model the instantaneous, dynamic response of the wing motion including the wing deformations, we extend the previous framework ${ }^{12}$ by adding a contribution from the forces due to the vortices in the flow field, such that

$$
F=F_{\mathrm{a}}+F_{\mathrm{v}}=\frac{\pi}{4} \rho_{\mathrm{f}} c^{2} \frac{d^{2} h}{d t^{2}}+\frac{1}{2} \rho_{\mathrm{f}} \frac{d h}{d t}\left|\frac{d h}{d t}\right| C_{\mathrm{v}} c,
$$

which is the Morison equation ${ }^{13}$ for the dimensional fluid dynamic force per unit length $F$. We model the added mass force $F_{\mathrm{a}}$ with the classic solution for the force acting normal to a thin flat plate with chord $c$ moving with $h(t)$. The second term $F_{\mathrm{v}}$ is the aerodynamic damping term which is proportional to the square of the wing velocity. The 
aerodynamic damping coefficient $C_{\mathrm{v}}$ depends on $k$, $R e$, etc. in general ${ }^{13}$ and may also be a function of space and time. Here, we approximate $C_{\mathrm{v}}$ as a constant to characterize its global impact on the wing deformation. Furthermore, we assume that the wing deformations relative to the imposed motion at the LE is small compared to the motion at the LE. As a result, we also assume that $F^{*}$ only depends on the imposed motion $h$ and we neglect the contribution of the passive pitch. The angular acceleration, velocity, as well as the angle itself can substantially contribute to the resulting fluid dynamic force ${ }^{14,19}$ when the wing deformations are significant and consequently change the response of the wing motion. For example is the aerodynamic damping term $(\mathrm{d} h / \mathrm{d} t)(\mathrm{d} w / \mathrm{d} t)$ can provide a first-order contribution, which arises when we replace the velocity of the imposed motion $\mathrm{d} h / \mathrm{d} t$ with the local velocity of the wing $\mathrm{d} v / \mathrm{d} t=\mathrm{d}(h+w) / \mathrm{d} t$ in Eq. (5). The investigation of the effects of these neglected terms is left as future study.

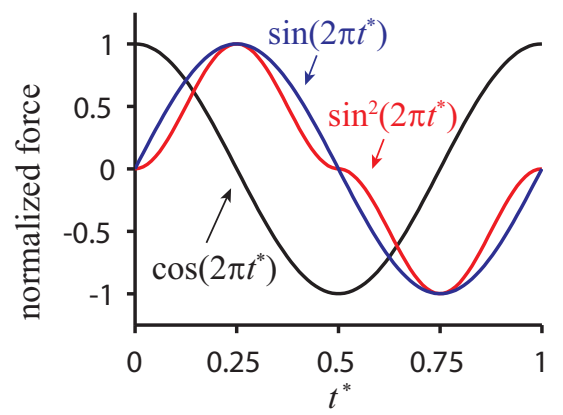

Figure 2. Three types of forces in Eq. (6) normalized by $\delta, \beta$, and $\zeta$, respectively as a function of $t^{*}$.

The solution of Eq. (3) with the fluid dynamics force term given by Eq. (5) can be obtained using the method of separation for the wing deformation relative to the imposed motion $w\left(x^{*}, t^{*}\right)=v\left(x^{*}, t^{*}\right)-h\left(t^{*}\right)=X\left(x^{*}\right) T\left(t^{*}\right)$ for the spatial component $X$ and the temporal component $T$. The solution procedure will not be detailed in this paper as it is straightforward and similar as in our previous investigation ${ }^{12}$. The solution of the spatial component is the same as for the classical vibration of a clamped beam with a free end ${ }^{12}$. The temporal part results in an ordinary differential equation for an oscillator for $0<t^{*} \leq 0.5$ as

$$
\frac{d^{2} T}{d t^{* 2}}+\zeta \frac{d T}{d t^{*}}+\omega_{f}^{2} T=\delta \cos \left(2 \pi t^{*}\right)+\beta \sin ^{2}\left(2 \pi t^{*}\right)+\zeta \sin \left(2 \pi t^{*}\right)
$$

where the non-dimensional damping factor is $\zeta=c / \Pi_{0}$ and the non-dimensional natural frequency is $\omega_{\mathrm{f}}^{2}=\left(2 \pi f_{1} / f\right)^{2}=k_{1}{ }^{4} \Pi_{1} / \Pi_{0}$ with $k_{1} \approx 1.875$ being the eigenvalue belonging to the first spatial mode $X_{1}$. In this study we only consider the effects of the first harmonic as it has been found that the most natural flyers operate at $f / f_{1}<0.8{ }^{17}$ and the first bending mode is the most dominant mode for the deformation of a flapping wing ${ }^{12,17,20}$. The first term in the RHS of Eq. (6) models the effects of the added mass and the inertial force of the wing, which are both acceleration related. The second term represents the aerodynamic damping term in Eq. (5), and the third term is due to the structural damping. Figure 2 shows that the acceleration related term has the highest contribution near the stroke-ends, while the aerodynamic damping term and the structural damping term peak at the midstroke. The coefficients $\delta$ and $\beta$ are defined as

$$
\delta=\frac{2 \pi^{2}\left(1+\frac{4}{\pi} \rho^{*} h_{s}^{*}\right) S t k}{\Pi_{0}}
$$

and

$$
\beta=\frac{C_{\mathrm{v}}}{\Pi_{0}}
$$

respectively.

The solution of Eq. (6) is 


$$
T\left(t^{*}\right)=T_{\text {transient }}+T_{\text {acceleration }}+T_{\text {aerodynamic }}+T_{\text {damping }}
$$

with

$$
\begin{aligned}
& T_{\text {transient }}=e^{-\frac{\zeta}{2} t^{*}}\left\{A \sin \left(\sqrt{\omega_{\mathrm{f}}^{2}-\left(\frac{\zeta}{2}\right)^{2}} t^{*}\right)+B \cos \left(\sqrt{\omega_{\mathrm{f}}^{2}-\left(\frac{\zeta}{2}\right)^{2}} t^{*}\right)\right\} \\
& T_{\text {acceleration }}=\delta \frac{\left[\left(-4 \pi^{2}+\omega_{\mathrm{f}}^{2}\right) \cos \left(2 \pi t^{*}\right)+2 \pi \zeta \sin \left(2 \pi t^{*}\right)\right]}{\left(-4 \pi^{2}+\omega_{\mathrm{f}}^{2}\right)^{2}+(2 \pi \zeta)^{2}} \\
& T_{\text {aerodynamic }}=\frac{\beta}{2}\left[\frac{1}{\omega_{\mathrm{f}}^{2}}-\frac{\left(-16 \pi^{2}+\omega_{f}^{2}\right) \cos \left(4 \pi t^{*}\right)+4 \pi \zeta \sin \left(4 \pi t^{*}\right)}{\left(-16 \pi^{2}+\omega_{\mathrm{f}}^{2}\right)^{2}+(4 \pi \zeta)^{2}}\right] \\
& T_{\text {damping }}=\frac{\zeta\left[-2 \pi \zeta \cos \left(2 \pi t^{*}\right)+\left(-4 \pi^{2}+\omega_{f}^{2}\right) \sin \left(2 \pi t^{*}\right)\right]}{\left(-4 \pi^{2}+\omega_{\mathrm{f}}^{2}\right)^{2}+(2 \pi \zeta)^{2}}
\end{aligned}
$$

where $A$ and $B$ are coefficients that can be determined from the initial conditions. Since the transient term $T_{\text {transient }}$ of Eq. (9) will be exponentially damped out, we will only discuss the effects of the remaining steady state terms.

The acceleration-related term $T_{\text {acceleration }}$ has the same origin as $\gamma$. For a system without structural damping, i.e. $\zeta=0$, the proportionality coefficient of this term becomes $\gamma=\delta /\left(4 \pi^{2}+\omega_{\mathrm{f}}^{2}\right)$. The effects of this term were discussed in detail in our previous work ${ }^{12}$.

The aerodynamic damping term $T_{\text {aerodynamic }}$ has two terms. The first term is inversely proportional to $\omega_{\mathrm{f}}^{2}$. Since $\omega_{\mathrm{f}}=2 \pi f_{1} / f, T_{\text {aerodynamic }}$ is proportional to the square of $f / f_{1}$. The second term has resonance behavior at $f / f_{1}=0.5$ for the undamped case, which suggests that the wing TE may substantially deflect when $f l f_{1} \approx 0.5$. It has been previously reported that the maximum propulsive force was obtained when $0.4<f / f_{1}<0.5^{21}$ or $0.5<f / f_{1}<0.7^{17}$. These findings suggest that there may be a strong correlation between the observed maximal propulsion and the resonance condition for the aerodynamic damping term. Here, we restrict the scope to the response of the wing dynamics and leave the discussion of the effects of the damping term on the aerodynamic performance to a future study.

The structural damping term $T_{\text {damping }}$ arises due to the imposed motion. This term has a phase shift of $\pi / 2$ with respect to the acceleration related term. This is not surprising as the velocity and the acceleration have a same phase shift of $\pi / 2$ for the considered harmonic motion, also shown in Eq. (6).

\section{B. Wing Deformation at the End-of-the-stroke}

It is known that the timing between translation and rotation is critical for lift generation. Specifically, when the wing rotated before it reaches the end-of-the-stroke, the highest lift coefficients are obtained ${ }^{15,22}$. This so-called advanced rotation performs better than the symmetric or the delayed rotational modes in which the wing reversal is synchronized or delayed with respect to the translation, respectively.

Despite the predicted optimal lift generation at the advanced rotation modes, hovering fruit flies ${ }^{10}$, honeybees ${ }^{16}$, beetles $^{5}$, and hymenopterans exhibit symmetric rotations in general ${ }^{5}$. The gap between the predicted maximum lift generation for advanced rotations and the symmetric rotations exhibited by a wide range of insects can be explained by considering the wing flexibility. Although tethered flying fruit flies actively control the wing rotation timings to initiate yaw turns ${ }^{2}$, these natural flyers possess flexible wings that deform significantly during flight that can enhance the aerodynamic performance ${ }^{4,23}$. Hovering flexible flapping wings with an imposed harmonic translational motion at the LE of the wing also generate passive pitch rotations with advanced, symmetric, and delayed rotational modes, where the phase lag strongly correlates to $f / f_{1}{ }^{14}$. More importantly, these flexible wings produce the highest lift for symmetric rotations, consistent with the reported observations of the aforementioned insects ${ }^{14}$.

For passive pitch motions, the resulting temporal evolution of the wing deformations can be multi-harmonic or even non-harmonic, contrary to the rigid wing cases where both motions are usually prescribed as harmonic functions ${ }^{15,22}$. To avoid ambiguity in the definition of phase lag, we consider the instantaneous passive pitch angle $\alpha_{e}$ due to the TE displacement relative to the LE at the stroke-end $\left(t^{*}=0.5\right)$ to determine the rotational mode. For example, when $\alpha_{\mathrm{e}}>90^{\circ}$, the wing TE advances to the imposed translation, which can be regarded as the advanced rotation mode. Similarly, at $\alpha_{\mathrm{e}}=90^{\circ}$ the passive rotation is symmetric and when $\alpha_{\mathrm{e}}<90^{\circ}$ the rotation is delayed.

Evaluating Eq. (9) at the stroke-end $t^{*}=0.5$ yields the relative TE deformation $w_{\mathrm{e}}$ as shown in Eq. (10). The deformation $w_{\mathrm{e}}$ has three components: the acceleration related term proportional to $\delta$; the aerodynamic damping 
term, proportional to $\beta$; and the structural damping term, proportional to $\zeta^{2}$. The aerodynamic damping term has two components, where the first component increases with $\left(f / f_{1}\right)^{2}$ and causes the wing to deform, such that the TE lags the LE. The second term has a relatively small contribution.

$$
\begin{gathered}
w_{\mathrm{e}}=\delta \frac{\left\{-1+\left(\frac{f_{1}}{f}\right)^{2}\right\}+\frac{\zeta^{2}}{2 \pi}}{4 \pi^{2}\left\{-1+\left(\frac{f_{1}}{f}\right)^{2}\right\}^{2}+\zeta^{2}}-\frac{\beta}{2}\left[\frac{1}{4 \pi^{2}}\left(\frac{f}{f_{1}}\right)^{2}-\frac{\left\{-4+\left(\frac{f_{1}}{f}\right)^{2}\right\}}{4 \pi^{2}\left\{-4+\left(\frac{f_{1}}{f}\right)^{2}\right\}^{2}+4 \zeta^{2}}\right] \\
-\frac{\frac{\zeta^{2}}{2 \pi}}{4 \pi^{2}\left\{-1+\left(\frac{f_{1}}{f}\right)^{2}\right\}^{2}+\zeta^{2}}
\end{gathered}
$$

Figure 3 shows the passive pitch angle $\alpha_{\mathrm{e}}=\arctan \left(w_{\mathrm{e}}\right)$ as a function of $f \mid f_{1}$. The unknown coefficients for the structural damping $c_{\mathrm{d}}$ and the aerodynamic damping $C_{\mathrm{v}}$ are empirically determined from the numerical data ${ }^{14}$ : $c_{\mathrm{d}}=0.65$ and $C_{\mathrm{v}}=0.75$ at constant reduced frequency of $k=0.6$. The correlation between Eq. (10) and the numerical data is good as shown in Fig. 3a. For lower frequency ratios $f / f_{1}<0.19, \alpha_{\mathrm{e}}$ increases and reaches the maximum of $\alpha_{\mathrm{e}}=100.6^{\circ}$ at $f / f_{1}=0.19$. In this range, the added mass term has the greatest contribution as illustrated in Fig. 3b, which shows the individual contributions of each term in Eq. (10). As $f / f_{1}$ increases further, the aerodynamic damping term starts to play a more dominant role. The rotational mode becomes symmetric at $f / f_{1}=0.32$ and decreases further into delayed rotational mode with $f / f_{1}$.

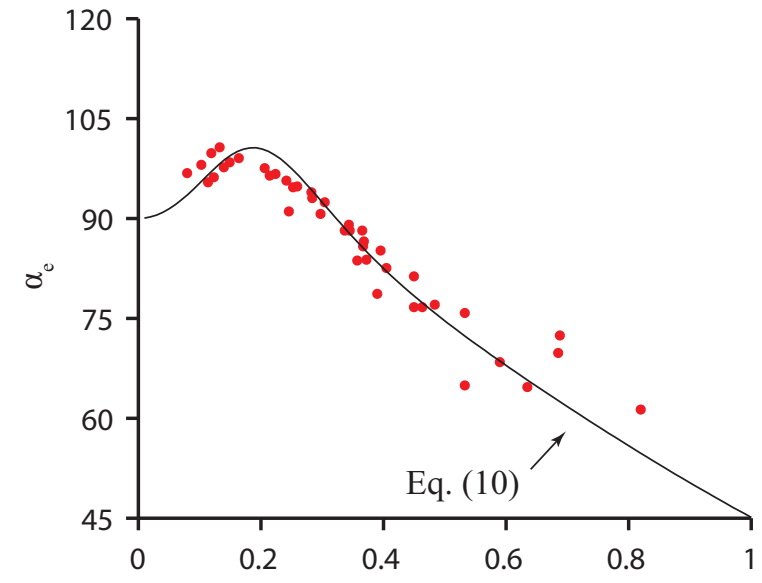

a)

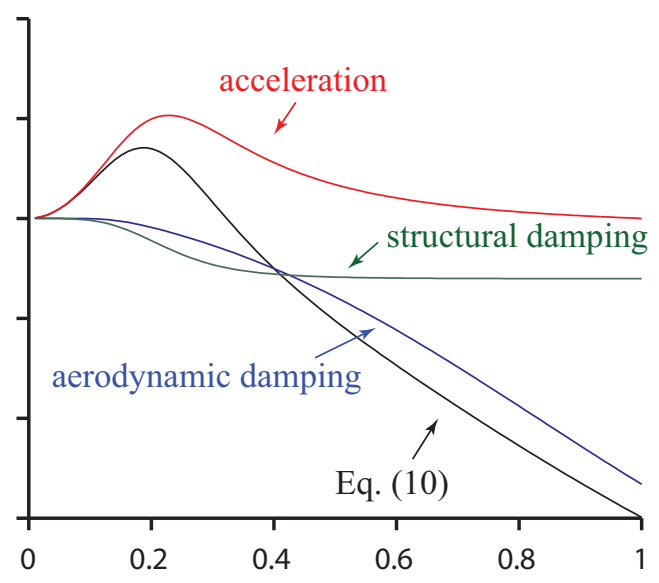

b)

$f / f_{1}$

Figure 3. Passive pitch angle $\alpha_{\mathrm{e}}$ at the end-of-the-stroke $\left(t^{*}=0.5\right)$. (a) The angle $\alpha_{\mathrm{e}}$ predicted by the current analysis (-) given by Eq. (10) is compared to the numerical data ( $\bullet$ obtained by Kang and Shyy ${ }^{14}$; (b) The total deformation (black) consists of the acceleration related term (red), aerodynamic damping term (blue), and structural damping term (green).

\section{Wing Deformation at the Midstroke}

Angle of attack is an essential measure in aerodynamics and it can directly affect leading-edge vortex generation and lift. For hovering rigid wings, flapping motions with a midstroke angle of attack of $40^{\circ}<\alpha_{\mathrm{m}}<50^{\circ}$ results in the highest lift generation in combination with advanced or symmetric rotations $\mathrm{s}^{22,24}$.

A prediction of the relative TE deformation $w_{\mathrm{m}}$ at the midstroke of a hovering flexible wing can be obtained from $w\left(t^{*}=0.25\right)$ in Eq. (9). The midstroke TE deformation $w_{\mathrm{m}}$ has the three components as shown in Eq. (11), where the aerodynamic damping term again has a term that is proportional to $\left(f l f_{1}\right)^{2}$. 


$$
\begin{gathered}
w_{\mathrm{m}}=\frac{\delta}{2 \pi} \frac{\zeta}{4 \pi^{2}\left\{-1+\left(\frac{f_{1}}{f}\right)^{2}\right\}^{2}+\zeta^{2}}+\frac{\beta}{2}\left[\frac{1}{4 \pi^{2}}\left(\frac{f}{f_{1}}\right)^{2}-\frac{\left\{-4+\left(\frac{f_{1}}{f}\right)^{2}\right\}}{4 \pi^{2}\left\{-4+\left(\frac{f_{1}}{f}\right)^{2}\right\}^{2}+4 \zeta^{2}}\right] \\
+\frac{\zeta\left\{-1+\left(\frac{f_{1}}{f}\right)^{2}\right\}}{4 \pi^{2}\left\{-1+\left(\frac{f_{1}}{f}\right)^{2}\right\}^{2}+\zeta^{2}}
\end{gathered}
$$

Figure $4 \mathrm{a}$ shows a comparison of $\alpha_{\mathrm{m}}$ based on Eq. (11) to the numerical data ${ }^{14}$. We assign the same values to the coefficients $C_{\mathrm{v}}, c_{\mathrm{d}}$, and $k$ as for $w_{\mathrm{e}}$ in Eq. (10). The numerical data show rather scattered trend, which suggests that $\alpha_{\mathrm{m}}$ only weakly correlates to $f / f_{1}$. For lower frequency ratios of $f l f_{1}<0.35$, the correlation between Eq. (11) and the numerical data is reasonable. The predicted trend is able to capture the increasing trend of the deflection, leading to an increased angle of attack. However, as the passive pitch motion transits into the delayed rotation mode near $f \mid f_{1} \approx 0.35$, see also Fig. 4 , the deformed wing orientation returns to the vertical shape. On the contrary, Eq. (11) decreases monotonically. Illustration of the three components shows that the acceleration related term is the foremost contributor to $\alpha_{\mathrm{m}}$. However, as $f / f_{1}$ increases further, the aerodynamic damping term determines the decreasing main trend, which is in the opposite direction to the tendency shown by the numerical data. A better fit that captures the reversing behavior of $\alpha_{\mathrm{m}}$ at $f / f_{1}>0.35$ is

$$
\alpha_{\mathrm{m}, \text { adj }}=\left\{\begin{array}{cc}
\alpha_{m}+5 f / f_{1}+3 & f / f_{1}<0.22 \\
\alpha_{m}-65 f / f_{1}+18.4 & f / f_{1} \geq 0.22
\end{array}\right.
$$

which is also shown in Fig. 4a.
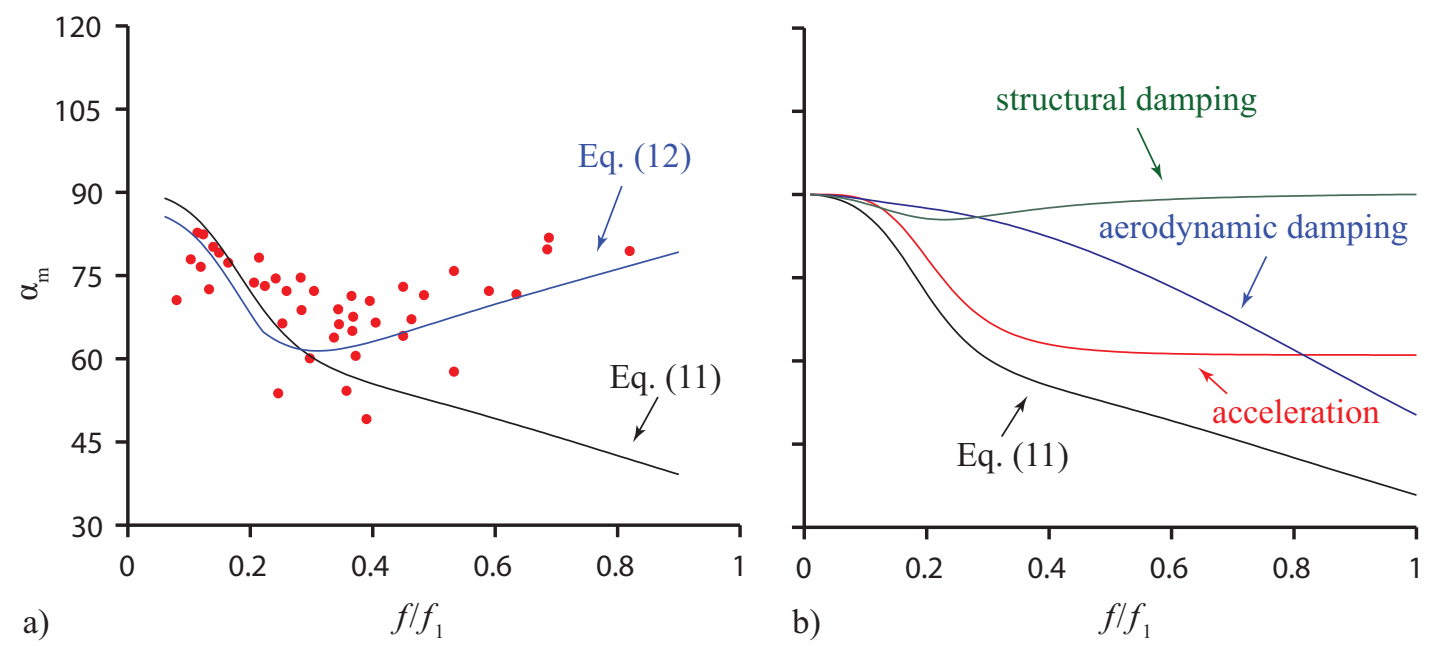

Figure 4. Passive pitch angle $\alpha_{\mathrm{m}}$ at the midstroke $\left(t^{*}=0.25\right)$. (a) The angle $\alpha_{\mathrm{m}}$ predicted by the current analysis $(-)$ given by Eq. (11) and its adjusted version (-) given by Eq. (12) are compared to the numerical data (•) obtained by Kang and Shyy ${ }^{14}$; (b) The total deformation (black) consists of the acceleration related term (red), aerodynamic damping term (blue), and structural damping term (green).

Potential reasons for the discrepancy can be found in the modeling of the fluid dynamic force terms in Eq. (5).

First, the coefficients $C_{\mathrm{v}}$ and $c_{\mathrm{d}}$ depend on the reduced frequency and the Reynolds number in general. Furthermore, these coefficients may also be a function of time and space. These coefficients are approximated as constants in this study.

Second, the incorporated fluid dynamic force terms are based on motions without pitching angles. The change of angle of attack due to wing deformations is not accounted for in Eq. (5). This may lead to errors especially near the 
midstroke where the translational velocity is at maximum. For example, empirically established quasi-steady models predict significant variations of the resulting force coefficients as a function of the angle of attack of the wing $\alpha$. Specifically, the drag force is $F_{\mathrm{d}}=0.5 \rho_{\mathrm{f}} U^{2} c C_{\mathrm{d}}$, where $C_{\mathrm{d}}=1.92-1.55 \cos (2.04 \alpha-9.82){ }^{22}$. Furthermore, we only model the fluid dynamic forces based on the imposed translational motion in Eq. (5). However, the local velocity of the wing has additional velocity component $\mathrm{d} w / \mathrm{d} t$, which may result in additional aerodynamic damping term $U \mathrm{~d} w / \mathrm{d} t$. Moreover, the passive pitch motion has an angular velocity $\mathrm{d} \alpha / \mathrm{d} t$ and acceleration $\mathrm{d}^{2} \alpha / \mathrm{d} t^{2}$, which contribute to the added mass as $\rho_{\mathrm{f}} \pi c^{2} U / 4 \mathrm{~d} \alpha / \mathrm{d} t$ and $-\rho_{\mathrm{f}} \pi c^{3} U / 8 \mathrm{~d}^{2} \alpha / \mathrm{d} t^{2}$, respectively ${ }^{14,19}$, where the pivot point is assumed to be at the LE. Also, the fluid dynamic force is assumed to be in the direction of motion, while the resulting force vector may rotate as the wing deforms and changes its orientation relative to the imposed motion. The magnitude of these terms depends on the translational velocity $U$. Since $U=0$ at the end-of-the-strokes, the influence from these terms on the resulting wing shape may be small at the end-of-the-strokes, which may explain the strong correlation in $w_{\mathrm{e}}$.

\section{Estimation of Time Evolution of Wing Deformation}

Approximations of the wing shapes are obtained at the end and midstrokes, by modeling the fluid dynamics force term using the Morison equation, Eq. (5), and approximating the wing dynamics using the beam equation, Eq. (3). While the prediction is reasonable for the midstroke passive pitch angle $\alpha_{\mathrm{m}}$ for $f / f_{1}<0.35$, the quality of the estimation deteriorates for higher $f / f_{1}$. The adjusted $\alpha_{\mathrm{m} \text {,adj }}$ gives a better representation of the qualitative behavior of the numerical data. On the other hand, the pitch angle at the end-of-the-stroke $\alpha_{e}$ correlates well with the reported data.

To estimate the instantaneous response of the wing motion, we use the angles $\alpha_{\mathrm{m}, \mathrm{adj}}$ and $\alpha_{\mathrm{e}}$ to construct a firstorder harmonic for the passive pitch $\alpha_{\mathrm{FH}}\left(t^{*}\right)$ as

$$
\alpha_{\mathrm{FH}}\left(t^{*}\right)=90-\alpha_{\mathrm{a}} \cos \left(2 \pi t^{*}+\varphi\right)
$$

where $\varphi$ is the phase lag between translation and rotation and $\alpha_{a}$ is the angular amplitude. The two unknowns $\varphi$ and $\alpha_{\mathrm{a}}$ are calculated by the two conditions $\alpha_{\mathrm{m}, \mathrm{adj}}=\alpha_{\mathrm{FH}}(0.25)$ and $\alpha_{\mathrm{e}}=\alpha(0)$, which yield a system of two equations for $\varphi$ and $\alpha_{\mathrm{a}}$.
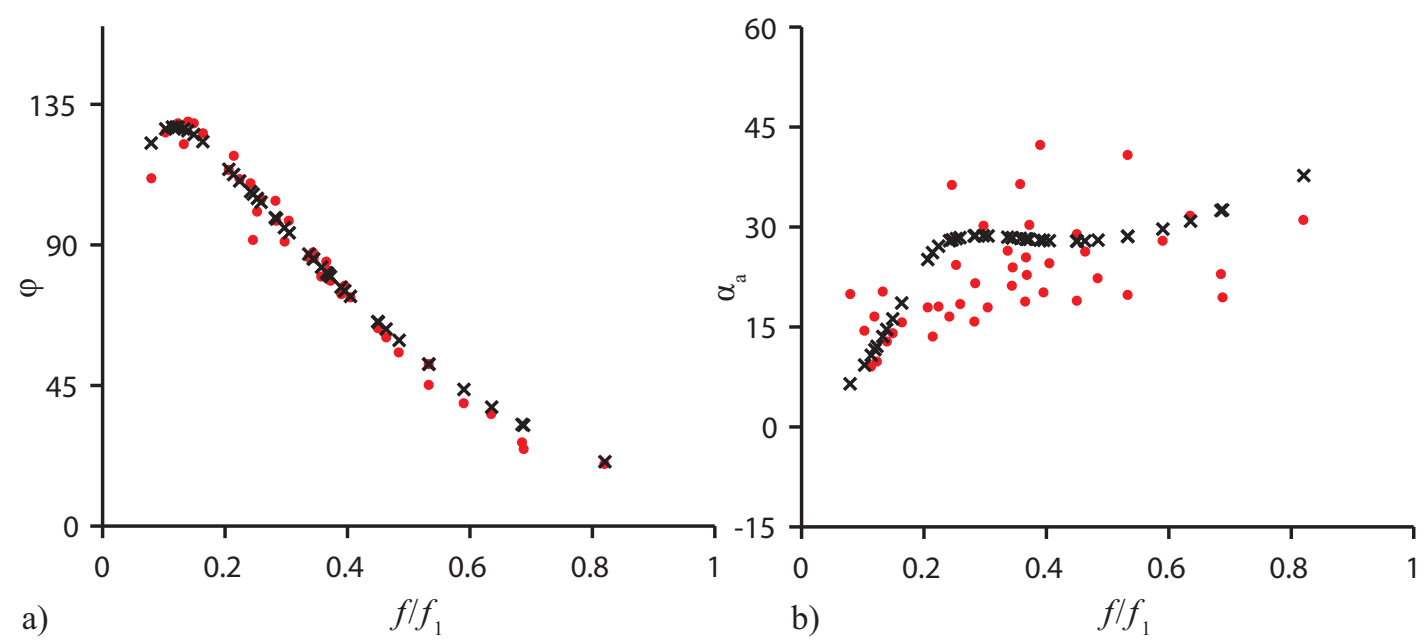

Figure 5. Characterization of the passive pitch based on a first-order harmonic representation of the passive pitch $\alpha_{\mathrm{FH}}$. (a) Phase lag $\varphi$ as a function of $f \mid f_{1}$. (b) Angular amplitude $\alpha_{\mathrm{a}}$ as a function of $f / f_{1} .(\times)$ Current analysis based on Eq. (13); (o) Numerical data obtained by Kang and Shyy ${ }^{14}$.

Figure 5 illustrates the phase lag $\varphi$ and the angular amplitude $\alpha_{a}$ based on Eq. (13). Similar to the relative TE deformation at the end-of-the-strokes, $\varphi$ increases for $f f f_{1}<0.14$. The motion of the TE in this frequency ratio range advances with respect to the imposed translation, resulting in an advanced rotation mode. After reaching the maximum $\varphi=132^{\circ}$ at $f / f_{1}<0.14, \varphi$ monotonically decreases to delayed rotation modes. When the passive wing rotation is in symmetric mode with the translation, the rotation lags behind the translation by $90^{\circ}$. Figure $5 \mathrm{a}$ shows 
that the symmetric rotation mode is reached around $f / f_{1} \approx 0.32$, corroborating the observation for $w_{\mathrm{e}}$ in Fig. 3 . Current analysis accurately predicts the phase lag obtained from the numerical data ${ }^{14}$. Both data set strongly correlates to $f / f_{1}$.

On the other hand, the angular amplitude $\alpha_{\mathrm{a}}$ calculated from the numerical data ${ }^{14}$ shows a more scattered behavior as a function of $f l f_{1}$, similar to the trend of $w_{\mathrm{m}}$. The predicted $\alpha_{\mathrm{a}}$ from the current study matches the increasing qualitative trend reasonably. Analysis of the numerical data shows that there exists a strong correlation between the maximum passive pitch during a stroke and $\gamma^{12}$, which will be investigated further in the future to model $\alpha_{\mathrm{m}}$ and $\alpha_{\mathrm{a}}$ more accurately.

Estimation of the time evolution of the wing deformation is presented in Fig. 6. With $\varphi$ and $\alpha_{a}$, the first-order harmonic representation of the passive pitch $\alpha_{\mathrm{FH}}$ can be determined as a function of time $t^{*}$ in Eq. (13). For the most cases, $\alpha_{\mathrm{FH}}\left(t^{*}\right)$ matches well with the time history of passive pitch that is determined from the numerical computations ${ }^{14}$. Overall, the estimation of the phase lag is accurate, but the estimation of the angular amplitude is reasonable for most cases. Figure 6 shows three representative cases for an advanced $\left(k=1.3, f l f_{1}=0.22\right)$, symmetric $\left(k=0.6, f \mid f_{1}=0.25\right)$, and delayed rotation $\left(k=3.05, f / f_{1}=0.48\right)$ mode. The time history of $\alpha$ for an advanced rotational mode is shown in Fig. 6a. The phase lag matches well with the numerical solution and the angular amplitude is reasonable. Figure $6 \mathrm{~b}$ shows the case with the largest difference in $\alpha\left(t^{*}\right)$. For this symmetric rotation case, the wing significantly deforms around the midstroke, resulting in a maximum passive pitch angle of $43^{\circ}$. While the phase lag is well predicted, the difference between the maximum angles becomes $20^{\circ}$. Note also that the numerical solution clearly consists of higher harmonic modes, while we only consider the first-order harmonic, which may have contributed to the observed difference. Finally, the predicted $\alpha_{\mathrm{FH}}$ for the delayed rotation case shown in Fig. $6 \mathrm{c}$ shows a close match in $\varphi$ and $\alpha_{a}$ with the numerical solution.
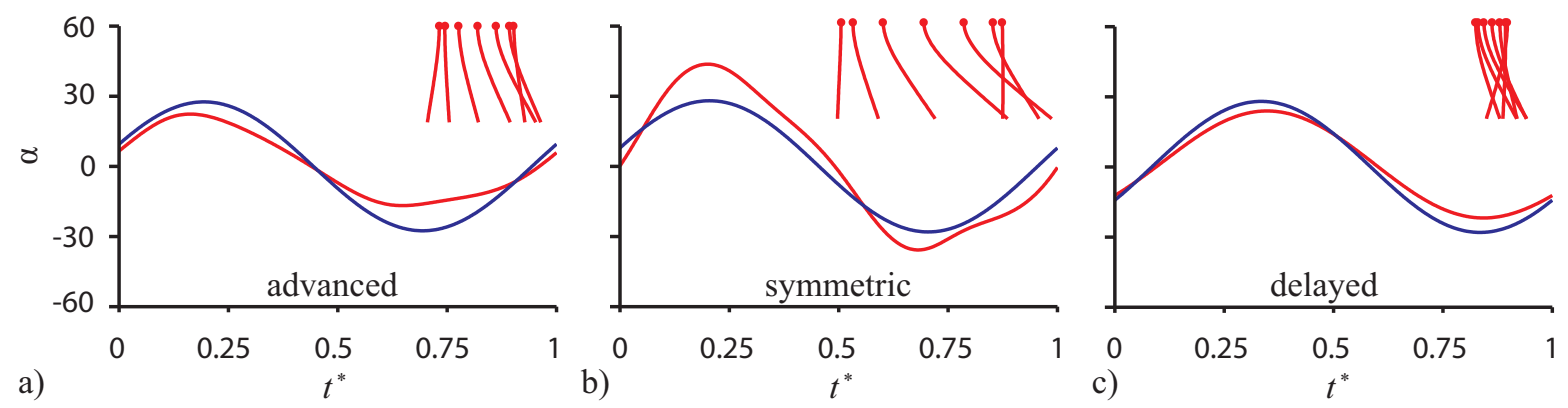

Figure 6. Instantaneous passive pitch $\alpha$ for an (a) advanced, (b) symmetric, and (c) delayed rotational mode. $(-)$ Current analysis using Eq. (13); (-) Numerical data obtained by Kang and Shyy ${ }^{14}$.

\section{Concluding Remarks}

This paper addresses modeling aspects of the response of the structural dynamics of a flapping, flexible wing at $R e=100$. We model the wing as a linear elastic flat plate and the fluid dynamics force with both added mass and aerodynamic damping forces using the Morison equation. The added mass force is proportional to the acceleration of the motion and the aerodynamic damping force depends on the square of the imposed translation.

The predicted passive pitch angle at the end-of-the-stroke shows a close agreement with the numerical solutions of a fully coupled aeroelastic framework. The end-of-the-stroke pitch angle is mainly caused by the added mass at lower frequency ratios, resulting in advanced rotation modes. As the frequency ratio increases, the aerodynamic damping term become more dominant, which works in the opposite direction of the added mass. As a result, the timing of the trailing-edge deformation relative to the leading-edge motion delays to the symmetric rotation and subsequently in delayed rotation modes.

The midstroke pitch angle only follows the trend of the numerical solutions for low frequency ratios and the predicted angle is not able to capture the reversing behavior at higher frequency ratios. This discrepancy may be solved with a more accurate modeling of the fluid dynamic force term.

We construct the instantaneous passive pitch based on the end-of-the-stroke and empirically corrected midstroke pitch angles as a first-order harmonic by calculating the phase lag and the angular amplitude. The predicted phase 
lag matched well with the numerical data with reasonable prediction of the angular amplitude. The estimated time evolution of the passive pitch agrees well with the numerical data.

In this study, we approximate the coefficients of the aerodynamic damping force term and the structural damping terms as constants. In principle, these coefficients are a function of space and time and depend on the reduced frequency, Reynolds number, etc. Moreover, we empirically determine the values by correlating the midstroke deformation to the numerical results. The reason is that the midstroke pitch angle weakly correlated to the frequency ratio, which suggests that another parameter may dictate the magnitude of the angle. Further analysis and modeling of these terms as well as uncovering the instantaneous aerodynamic performance based on the current results are left as a future study.

Nevertheless, we describe a model of how the passive pitch due to wing deformations evolves in time. This result is promising and may be highly relevant toward an accurate modeling of aerodynamic force on a flexible flapping wing as a function of time. Such derivation of instantaneous aerodynamic force can be useful to understand the complex, but intriguing physics of flexible flapping wings and help the development of Micro-Air Vehicles.

\section{References}

${ }^{1}$ Shyy, W., Lian, Y., Tang, J., Viieru, D., and Liu, H., Aerodynamics of Low Reynolds Number Flyers, Cambridge Univ Press, New York, USA, 2008.

${ }^{2}$ Dickinson, M. H., Lehmann, F. O., and Götz, K. G., "The Active Control of Wing Rotation by Drosophila," The Journal of Experimental Biology, Vol. 182, 1993, pp. 173-89.

${ }^{3}$ Lee, J.-S., Kim, J.-K., Kim, D.-K., and Han, J.-H., "Longitudinal Flight Dynamics of Bioinspired Ornithopter Considering Fluid-Structure Interaction," Journal of Guidance, Control, and Dynamics, Vol. 34, No. 3, 2011, pp. 667-677.

${ }^{4}$ Young, J., Walker, S. M., Bomphrey, R. J., Taylor, G. K., and Thomas, A. L. R., "Details of Insect Wing Design and Deformation Enhance Aerodynamic Function and Flight Efficiency," Science, Vol. 325, No. 5947, 2009, pp. 1549-52.

${ }^{5}$ Lehmann, F.-O., Gorb, S., Nasir, N., and Schützner, P., "Elastic Deformation and Energy Loss of Flapping Fly Wings," Journal of Experimental Biology, Vol. 214, No. 17, 2011, pp. 2949-61.

${ }^{6}$ Curet, O. M., Swartz, S. M., and Breuer, K. S., "An aeroelastic instability provides a possible basis for the transition from gliding to flapping flight.," Journal of the Royal Society, Interface / the Royal Society, Vol. 10, No. 80, 2013, p. 20120940.

${ }^{7}$ Alben, S., Shelley, M., and Zhang, J., "Drag Reduction through Self-similar Bending of a Flexible body," Nature, Vol. 420, No. 6915, 2002, pp. 479-481.

${ }^{8}$ Shelley, M. J., and Zhang, J., "Flapping and Bending Bodies Interacting with Fluid Flows," Annual Review of Fluid Mechanics, Vol. 43, No. 1, 2011, pp. 449-465.

${ }^{9}$ Nakata, T., and Liu, H., "Aerodynamic Performance of a Hovering Hawkmoth with Flexible Wings: a Computational Approach,” Proceedings of the Royal Society B, Vol. 279, No. 1729, 2012, pp. 722-731.

${ }^{10}$ Fry, S. N., Sayaman, R., and Dickinson, M. H., "The Aerodynamics of Hovering Flight in Drosophila.," Journal of Experimental Biology, Vol. 208, No. 12, 2005, pp. 2303-2318.

${ }^{11}$ Mountcastle, A. M., and Combes, S. A., "Wing Flexibility Enhances Load-lifting Capacity in Bumblebees," Proceedings of the Royal Society B, Vol. 280, No. 1759, 2013, pp. 20130531-20130531.

${ }^{12}$ Kang, C.-K., Aono, H., Cesnik, C. E. S., and Shyy, W., "Effects of Flexibility on the Aerodynamic Performance of Flapping Wings," Journal of Fluid Mechanics, Vol. 689, 2011, pp. 32-74.

${ }^{13}$ Shih, C. C., and Buchanan, H. J., "The Drag on Oscillating Flat Plates in Liquids at Low Reynolds Numbers," Journal of Fluid Mechanics, Vol. 48, No. 2, 1971, pp. 229-239.

${ }^{14}$ Kang, C.-K., and Shyy, W., "Scaling Law and Enhancement of Lift Generation of an Insect-size Hovering Flexible Wing," Journal of Royal Society Interface, in press.

${ }^{15}$ Trizila, P., Kang, C.-K., Aono, H., Shyy, W., and Visbal, M., "Low-Reynolds-Number Aerodynamics of a Flapping Rigid Flat Plate," AIAA Journal, Vol. 49, No. 4, 2011, pp. 806-823.

${ }^{16}$ Altshuler, D. L., Dickson, W. B., Vance, J. T., Roberts, S. P., and Dickinson, M. H., "Short-amplitude Highfrequency Wing Strokes Determine the Aerodynamics of Honeybee Flight," Proceedings of the National Academy of Sciences of the United States of America, Vol. 102, No. 50, 2005, pp. 18213-18218.

${ }^{17}$ Ramananarivo, S., Godoy-Diana, R., and Thiria, B., "Rather than Resonance, Flapping Wing Flyers May Play on Aerodynamics to Improve Performance," Proceedings of the National Academy of Sciences of the United States of America, Vol. 108, No. 15, 2011, pp. 5964-5969. 
${ }^{18}$ Saffman, P. G., Vortex Dynamics, Cambridge University Press, 1995.

${ }^{19}$ Sane, S., and Dickinson, M., "The Aerodynamic Effects of Wing Rotation and a Revised Quasi-steady Model of Flapping Flight," Journal of Experimental Biology, Vol. 205, No. 8, 2002, pp. 1087-1096.

${ }^{20}$ Ferreira de Sousa, P. J. S. a., Allen, J. J., and De Sousa, P. J. S. A. F., "Thrust Efficiency of Harmonically Oscillating Flexible Flat Plates," Journal of Fluid Mechanics, Vol. 674, 2011, pp. 43-66.

${ }^{21}$ Yin, B., and Luo, H., "Effect of Wing Inertia on Hovering Performance of Flexible Flapping Wings," Physics of Fluids, Vol. 22, 2010, p. 111902.

${ }^{22}$ Dickinson, M. H., Lehmann, F. O., and Sane, S. P., "Wing Rotation and the Aerodynamic Basis of Insect Flight," Science, Vol. 284, No. 5422, 1999, pp. 1954-1960.

${ }^{23}$ Walker, S. M., Thomas, A. L. R., and Taylor, G. K., "Deformable Wing Kinematics in the Desert Locust: How and Why Do Camber, Twist and Topography Vary through the Stroke?," Journal of the Royal Society, Interface, Vol. 6, No. 38, 2009, pp. 735-747.

${ }^{24}$ Sane, S., and Dickinson, M., "The Control of Flight Force by a Flapping Wing: Lift and Drag Production," Journal of Experimental Biology, Vol. 204, No. 15, 2001, pp. 2607-2626. 